Apidologie, 1972, 3 (1), 55-78.

\title{
DER EINFLUSS VERSCHIEDENER KONSTITUTIONS- UND UMWELTFAKTOREN AUF DIE ANFÄLLIGKEIT DER HONIG- BIENE (APIS MELLIFICA L.) GEGENÜBER ZWEI INSEKTIZIDEN PFLANZENSCHUTZMITTELN *
}

\author{
L'influence de différents facteurs internes et externes \\ sur la sensibilité des abeilles (Apis mellifica $L$.) \\ à l'égard de deux produits phytosanitaires insecticides
}

\author{
Anastasios LADAS \\ Institut für Landwirtschaftiche \\ Zoologie und Bienenkunde der \\ Universität Bonn
}

\begin{abstract}
SUMMARY
THE INFLUENCE OF SOME INTERNAL AND EXTERNAL FACTORS UPON THE INSECTICIDE RESISTA NCE OF HONEYBEE
\end{abstract}

The following influences upon insecticide resistance of honey bees were tested : age, race, degree of inbreeding, application in light and darkness and Nosema infection. The applied insecticides were DiDiTan (DDT) for contact and the organophosphate Dipterex for oral effect.

A new method for the application of the contact insecticide was developed while for the oral application a standard laboratory method was used.

Young bees proved less resistant to DDT than older ones in $22^{\circ} \mathrm{C}$ as well as in $35^{\circ} \mathrm{C}$.

The Caucasian bees showed lesser susceptibility to $D D T$ and Dipterex than bees of the Carniolan and Italian race. Between Carniolan bees of different geographical origin significant differences in resistance could also be observed.

Two inbred Carniolan lines $(F=0,25)$ reacted differently to $D D T$. In the hybrids of these two lines $(F=0,03)$, however, no difference could be found in comparison to the paternal line, but they proved more resistant than bees of the maternal line. In contact as well as

* Für die Ủberlassung des Themas sowie das Interesse am Fortgang der Untersuchungen danke ich Herrn Dr. Drescher, ebenso allen Institutsmitgliedern, die mir bei den experimentellen Arbeiten behilflich waren. Herr Prof. Dr. Wurmbach ermöglichte mir die Durchführung der Arbeit im Institut für Landwirtschaftliche Zoologie und Bienenkunde, Universität Bonn. 
in feeding experiments the keeping of the bees in darkness resulted in an increased toxicity of the insecticides. The effects after keeping in darkness and light are significant. - Carniolan bees artificially infected with Nosema spores proved more susceptible to $D D T$ and Dipterex than healthy bees.

$D D T$ shows a negative temperature coefficient, which means that with decreasing temperature the effect of the insecticide increases. The effect of Dipterex is less in fluenced by temperature, only a minor positive temperatur coefficient could be demonstrated. The pH-value of the Dipterex solution was of great in fluence on toxicity.

\section{ZUSAMMENFASSUNG}

Der Einfluß der Faktoren Alter, Rasse, Inzuchtgrad, Applikation bei Licht - und Dunkelhaltung und Nosemainfektion auf die Wirkung des Kontaktgiftes DiDiTan Ultra (DDT-Wirkstoff) und des Fraßgiftes Dipterex (Phosphorsäureester) bei Bienen wurde geprüft. Zur Ermittlung des Kontaktgiftwertes von DDT konnte eine neue Applikationsmethode entwickelt werden. Die Bestimmung des peroralen Toxizitätsgrades von Dipterex SP 80 erfolgte mit einer standardisierten Laboratoriumsmethode.

Sowohl bei $22^{\circ} \mathrm{C}$ als auch bei $35^{\circ} \mathrm{C}$ zeigten sich Jungbienen empfindlicher gegenüber DiDiTan Ultra als alte Bienen.

Die Caucasica-Bienen erwiesen sich als widerstandsfähiger gegenüber DiDiTan Ultra und Dipterex SP 80 im Vergleich mit Bienen der Carnica - und Ligustica-Rasse. Statistisch gesicherte Verträglichkeitsunterschiede gegenüber DiDiTan Ultra konnten auch bei Bienen verschiedener CarnicaHerkünfte festgestellt werden.

Zwei ingezüchtete Linien (Carnica, $F=0,25$ ) reagierten gegenüber DiDiTan Ultra unterschiedlich. Bienen der $F_{1}$-Generation $(F=0,03)$ dagegen zeigten keinen Unterschied gegenüber den Bienen des Vatervolkes: sie waren aber widerstandsfähiger als die Tiere des Muttervolkes.

Sowohl bei Kontakt- (DiDiTan Ultra) als auch bei Fraßversuchen (Dipterex SP 80) steigerte Dunkelheit die Wirkung der geprüften Insektizidpräparate. Der Unterschied zwischen der Bienenreaktion bei Licht- und Dunkelhaltung ist signifikant.

Mit Nosemasporen künstlich infizierte Bienen (Carnica) waren anfälliger gegenüber DiDiTan Ultra und Dipterex SP 80 als nicht infizierte.

DDT besitzt einen negativen Temperaturkoeffizienten, d.h. die Wirksamkeit des Giftes nimmt bei sinkender Temperatur zu.

Dipterex SP 80 ist weniger temperaturabhängig als DiDiTan Ultra. Es besitzt einen positiven Temperaturkoeffizienten.

Als ausschlaggebend für den Toxizitätsgrad von Dipterex SP 80 erwies sich der pH-Wert bzw. das Alter der jeweils zur Anwendung kommenden Giftlösung. Im alkalischen wässrigen Bereich ist der Giftwert von Dipterex $S P 80$ größer als im sauren.

\section{I. - EINLEITUNG}

Der verbreitete Einsatz chemischer Stoffe zur Bekämpfung landwirtschaftlicher Schadinsekten erfordert die Prüfung der in den Handel gebrachten Präparate auf ihre Toxizität gegenüber der Honigbiene.

Zur Durchführung dieser Prüfung steht eine standardisierte Laboratoriumsmethode zur Verfügung (STUTE, 1969). Bei den verwendeten Bienen lassen sich jedoch in einigen Fällen merkliche Unterschiede in der Reaktion auf die Insektizide bei gleicher Applikationsform feststellen (Maurizio und Schenker, 1957 ; Anderson und Atkins, 1958 ; Beran, 1958 ; КосH, 1959 ; Atkins und Anderson, 1962 ; Graves und Mackensen, 1965).

Aufgabe dieser Untersuchung war es, den Einfluß verschiedener konstitutioneller und konditioneller Faktoren - Alter, Rasse bzw. Herkunft, 
Inzuchtgrad, Applikation bei Licht und Dunkelheit und Nosemainfektion auf die toxische Wirkung von zwei Insektiziden (DDT und ( Dipterex ») auf Bienen zu untersuchen.

Die beiden Präparate wurden aus zwei verschiedenen Insektizidkategorien gewählt, um eventuell vorhandene multiple Resistenz bestimmter Bienenherkünfte zu prüfen (Atkins und Anderson, 1962 ; Graves und MackenSEN, 1965).

Zur Durchführung der Versuche entwickelte ich eine eigene Methode der Insektizidapplikation und der Haltung der Versuchstiere.

\section{II. - MATERIAL UND METHODE}

\section{a. - Insektizide und Bienenmaterial}

Insektizide : Für die Versuche habe ich die Handelspräparate DiDiTan Ultra (Schering AG - Berlin) und Dipterex SP 80 (Farbenfabriken BAYER AG - Leverkusen) benutzt. Die Packungen wurden während der zweijährigen Versuchsdauer im Kühlschrank bei $3-6^{\circ} \mathrm{C}$ luftdicht aufbewahrt.

DiDiTan Ultra enthält $80 \%$ Dichlor-diphenyl-trichloräthan (DDT). Das Präparat wirkt als Kontakt- und Fraßgift und besitzt langanhaltende Wirkung. Die für die Praxis empfohlenen Konzentrationen schwanken je nach Anwendungsbereich zwischen 0,1 - $1 \%$.

Dipterex SP 80 ist ein $80 \%$ iges Phosphorsäureester-Präparat. Es wirkt vor allem als Magengift. Der Wirkstoff besitzt ausserdem eine Tiefen- und systemische Wirkung (UnTERsTENhöFER, 1957, 1958; Unterstengöfer und FrehSe, 1963). Sein Toxizitätsgrad ist stark vom $\mathrm{pH}$-Wert und von der Temperatur des Lösungsmittels abhängig (Schrader, 1963; UNTERSTENhöFER und FrehSE, 1963). Die Halbwertszeiten von Dipterex betragen in wässriger Lösung von ca. $20^{\circ} \mathrm{C}$ bei $\mathrm{pH}=8,063$ Minuten, bei $\mathrm{pH}=7,0386$ Minuten und bei $\mathrm{pH}=6,089$ Stunden. Die Umsetzungsquote ist bei $\mathrm{pH}=5,4$ sehr niedrig (MÜhlmanN und Schrader, 1957; Metcalf et al., 1959; Fucuto, 1961; Schrader, 1963).

Die Konzentration des Präparates Dipterex SP 80 beträgt in der landwirtschaftlichen Praxis 0,1 bis $0,15 \%$.

Bienenmaterial : Für die Versuche dienten Bienen (Apis mellifica L.) verschiedener Rassen bzw. Herkünfte (Tab. 1).

Ich arbeitete mit Sommer- und Winterbienen. Verdeckelte Brutwaben entnahm ich den entsprechenden Bienenvölkern, ließ die Arbeiterinnen in einem Brutschrank schlüpfen, markierte die frischgeschlüpften Bienen auf dem Thorax und gab sie in ein Pflegevolk. Diese Art der Behandlung lieferte altersmäßig definierte Bienen in adäquater physiologischer Verfassung.

Geringe Abweichungen von dieser Behandlung erforderte die Prüfung der mit Nosemasporen infizierten Bienen. 18 bis 25 Tage alte Bienen entnahm ich dem Pflegevolk und infizierte sie im Laboratorium mit Nosemasporen. Jeweils $10 \mathrm{mg}$ Sporenpräparat (aus an Nosema erkrankten Bienen gewonnen* und ca. 30 Tage bei Zimmertemperatur aufbewahrt) wurden mit $2 \mathrm{ml}$ einer $50 \%$ igen Zuckerlösung gemischt. Aus dieser Sporenaufschwemmung erhielt jede Biene $10 \mathrm{~mm}^{3}$ (das entspricht ca. $400000-500000$ Sporen / Biene). Anschließend wurden 30 bis 40 infizierte Tiere in Holzkästchen (Volumen ca. $150 \mathrm{~mm}^{3}$ ) 6,7 und 8 Tage im Brutschrank bei ca. $32^{\circ} \mathrm{C}$ gehalten (Goetze et al., 1959) und während dieser Zeit mit $50 \%$ igem Zuckerwasser gefüttert. Die Kontrollbienen erfuhren die gleiche Behandlung. Nach dieser Vorbehandlung applizierte ich die beiden Insektizide bei beiden Gruppen.

Im AnschluB an die Versuche erfolgte eine lichtmikroskopische Untersuchung der toten und lebenden Bienen zur Bestimmung des Infektionsgrades. Dazu wurde das Abdomen jeder Biene isoliert und in etwa $0,5 \mathrm{ml}$ Wasser zerquetscht. Die Befunde aus jedem Versuch sind in Prozent dargestellt. 
TAB. 1. - Rassen und Herkünfte der Versuchsbienen

TABL. 1. - Race et origine des abeilles utilisêes pour les essais

\begin{tabular}{|c|c|}
\hline $\begin{array}{l}\text { Rasse } \\
\text { Race }\end{array}$ & $\begin{array}{c}\text { Herkunft } \\
\text { Origine }\end{array}$ \\
\hline a) Carnica : & $\begin{array}{l}\text { 1. Trifolium (langjährig. Zuchtstamm des Inst. f. Landw. Zoologie-u. } \\
\text { Bienenkunde) } \\
\text { Trifolium (souche entretenue depuis de longues années par l'Institut de Zoologie agrico } \\
\text { et d'apiculture) } \\
\text { 2. Mazedonien. Macédoine.* } \\
\text { 3. Osterreich. Autriche.* } \\
\text { 4. Ungarn. Hongrie.* }\end{array}$ \\
\hline $\begin{array}{l}\text { b) Carnica : } \\
\text { (Inzucht) } \\
\text { (Consanguine) }\end{array}$ & 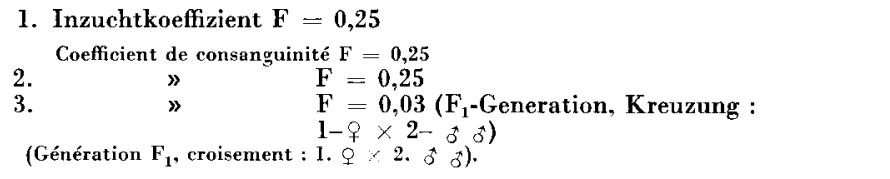 \\
\hline c) Ligustica : & $\begin{array}{l}\text { Züchter PIANA (Bologna). } \\
\text { Élevage PIANA (Bologne) }\end{array}$ \\
\hline d) Caucasica : & $\begin{array}{l}\text { Importiertes Material aus Russland. } \\
\text { Matériel importé de Russie. }\end{array}$ \\
\hline
\end{tabular}

* Nachkommen von importierten Original-Königinnen.

Descendants de reines d'origine importées.

** Herkunft der ingezüchteten Bienenlinien $(1,2)$ aus einem Ausgangsvolk. Origine des lignées consanguines $(1,2)$ à partir d'une colonie.

Außerdem prüfte ich die Wirkung beider Präparate auf Bienenarbeiterinnen unter den Bedingungen der Licht- und Dunkelhaltung in zwei Brutschränken gleicher Bauart bei gleicher Temperatur $\left(28^{\circ} \mathrm{C}\right.$ ). Zur Beleuchtung diente eine in etwa $20 \mathrm{~cm}$ Abstand von den Versuchstieren im Brutschrank angebrachte Glühbirne $(25 \mathrm{~W}$ att $)$.

\section{b. - Prüfmethoden}

Herstellung der Versuchskäfige zur Bestimmung der Kontaktgiftwirkung : Das Präparat löste ich in reinem Azeton. Ein optimal gleichmäßiger Film des gelösten Insektizides auf einer Cellophanfolie (Zellglas No 305, Fa. Hetzel \& Co. - Stuttg.) konnte durch folgendes Spritzverfahren erzielt werden : $0,2 \mathrm{ml}$ Flüssigkeit wurden mit einer geeichten Pipette in eine Glasdüse eingeführt. Der Abstand der Düsenspitze von einem ca. $15 \times 15 \mathrm{~cm}$ messenden gestanzten Cellophanblättchen betrug etwa $4,5 \mathrm{~cm}$. Das Cellophanblatt lag auf einer drehbaren Scheibe. Der Fußlotpunkt der Düse auf dem Cellophan bzw. der Drehscheibe war ca. 1,5 cm vom Mittelpunkt der Scheibe entfernt. Die Scheibe drehte sich mit 78 Umdreh./Minute während ein Preßluftstrom (unter 1 at) durch den Zerstäuber geleitet wurde. Nach dem Öffnen des kleinen Hahnes der Glasdüse war in 4-5 Sekunden die gesamte Flüssigkeit appliziert (Abb. 1). Der aufgesprühte Film bedeckte einen kreisförmigen Teil des Blattes von etwa $7 \mathrm{~cm}$ Durchmesser (Abb. 2), wobei keine wesentliche Schrumpfung des Cellophans erfolgte. Sprühverluste traten bei dieser Methode nicht in nachweislichem Umfang auf.

Jeweils zwei der so behandelten Cellophanscheiben bildeten Boden und Deckel von flachen zylindrischen Käfigen (Volumen ca. $45 \mathrm{~cm}^{3}$, Gesamtober fläche ca. $100 \mathrm{~cm}^{2}$, behandelte Fläche ca. $75 \mathrm{~cm}^{2}$ ). Der große Anteil der mit dem Präparat bedeckten Cellophanfläche garantierte einen intensiven Kontakt mit den Tieren. Für die ringförmige Seitenfläche (Peripherie) der 


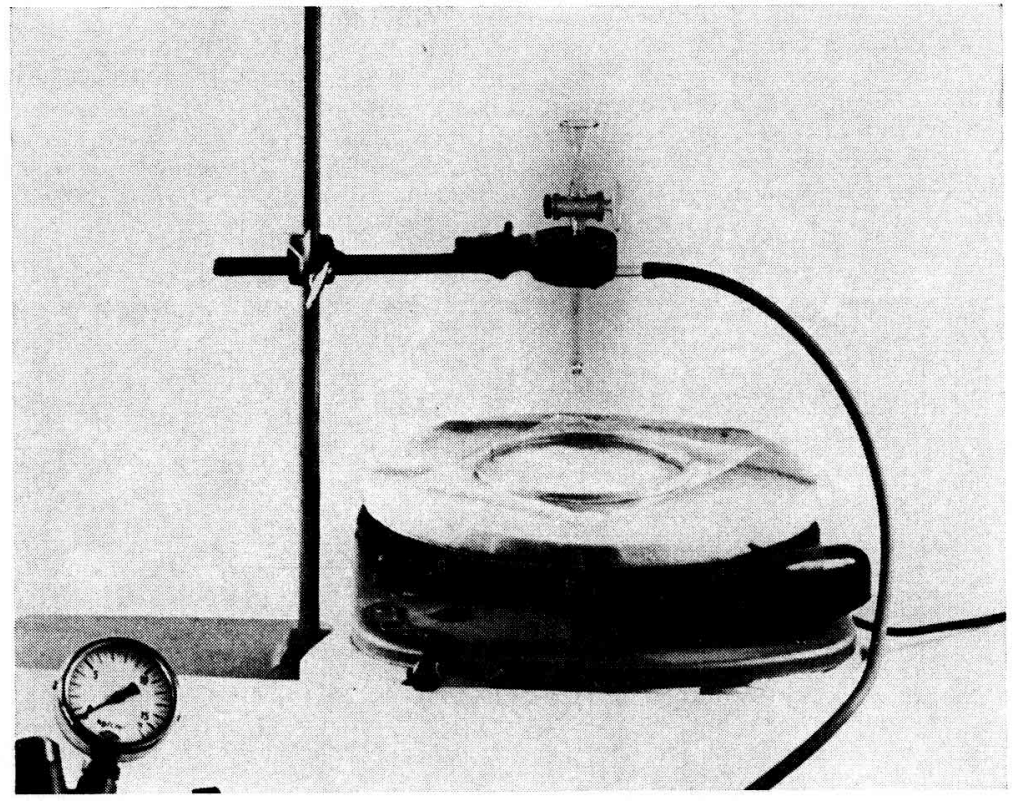

Aвв. 1. - Apparatur zur Giftverteilung

Fig. 1. - Appareil pour la répartition du toxique

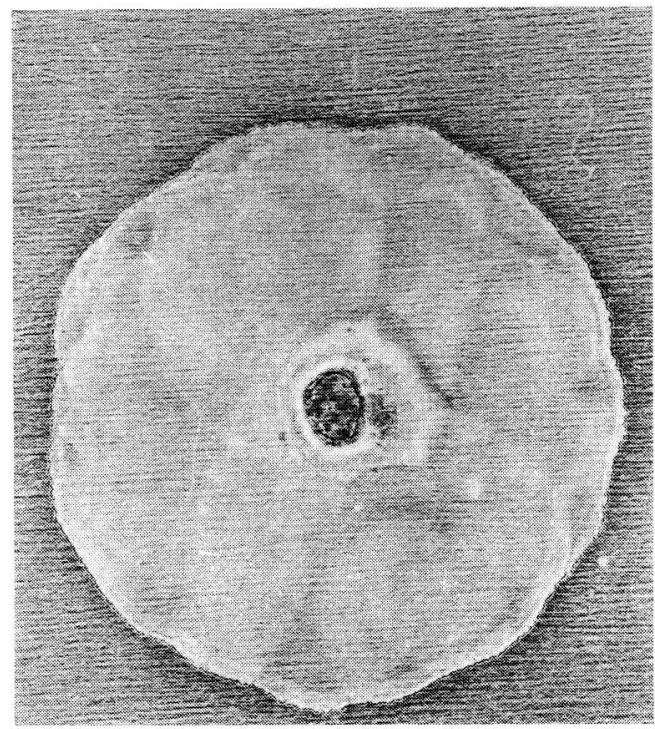

Aв8. 2. - DiDiTan-Ultra-Filmbildung auf Cellophan

FIg. 2. - Formation du film de DiDiTan-Ultra sur la cellophane 
Käfige fanden gleichmäßig (mit Hilfe einer Kreissäge) geschnittene Ringe von ca. $11 \mathrm{~mm}$ Höhe aus einem Plastikab flußrohr Verwendung $(70 \mathrm{~mm}$ Nennweite; Fa. Kunststoffwerk Gebr. Anger GmbH \& Co. - München). In jeden Ring bohrte ich zur Bieneneinbringung und -fütterung eine Öffnung von $8 \mathrm{~mm}$ Durchmesser. Ein zentral gelochtes Korkstück diente dem Verschluß der Öffnung sowie dem Festhalten des pipettenförmigen Futterröhrchens $(80 \mathrm{~mm}$ lang, $5 \mathrm{~mm}$ Durchmesser). Infolge der etwas nach oben gerichteten Stellung der Pipette konnte die zur Fütterung verwendete $50 \%$ ige Zuckerlösung nicht herausfliessen (Abb. 3 und 4). Diese Anordnung ermöglichte eine Futterversorgung ohne Kontamination durch das zu prüfende Mittel.

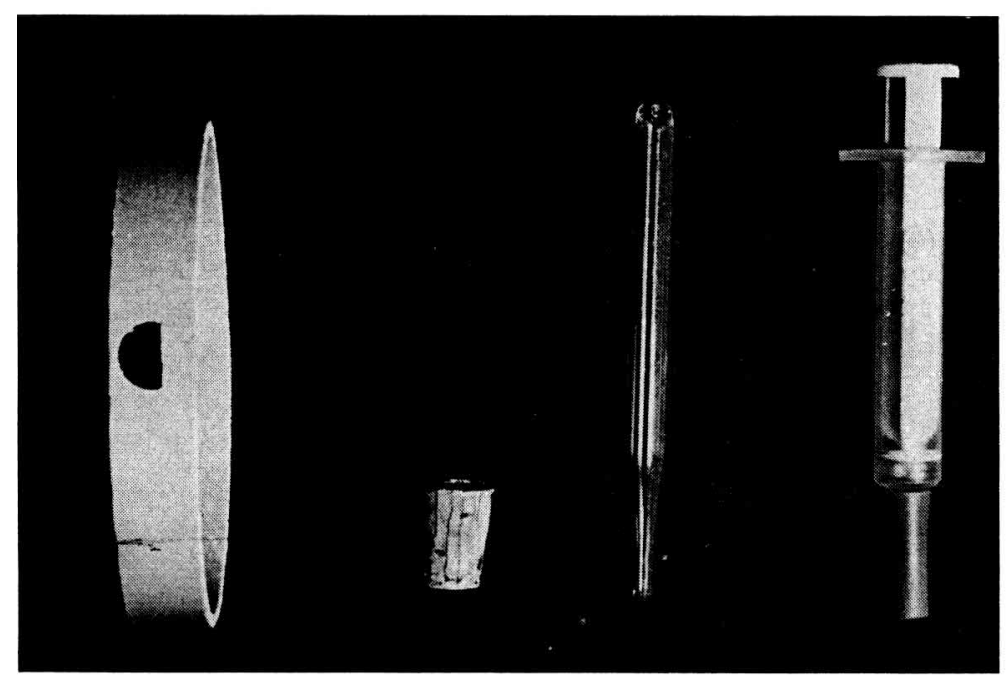

Aв8. 3. - Kunststoffring mit Futtereinrichtung

FIG. 3. - Anneau de matière plastique avec dispositif pour le nourrissement

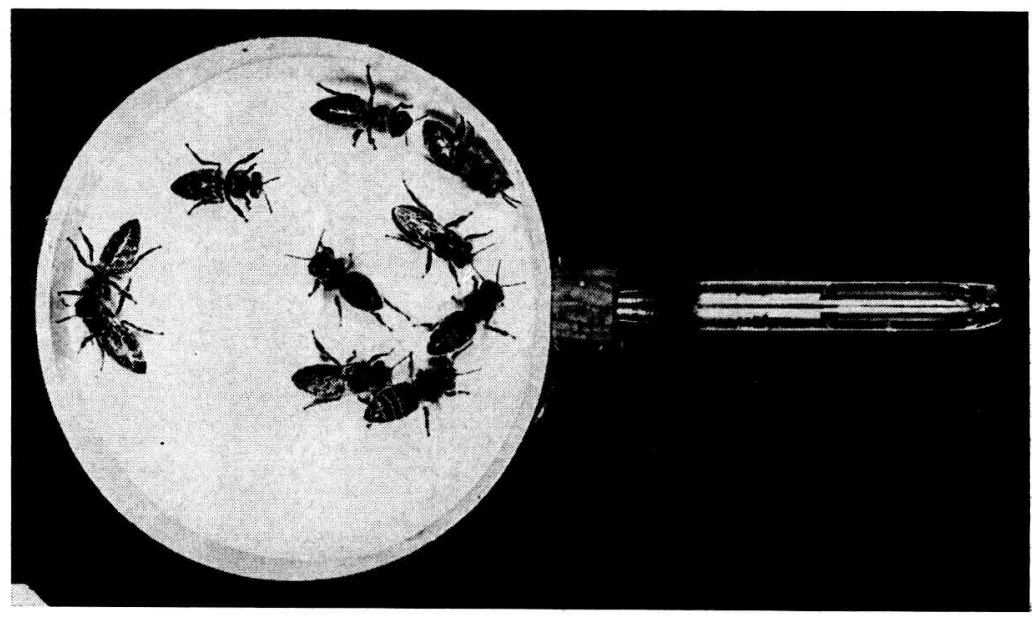

Aв8. 4. - Cellophankäfig besetzt mit Bienen und Fütterungsanlage

FIG. 4. - Cage de cellophane occupée par les abeilles et dispositif pour le nourrissement 


\section{ANFÄLLIGKEIT GEGENÜBER ZWEI INSEKTIZIDEN}

Zur Befestigung der Cellophanscheiben am Ring brachte folgende Methode Erleichterung und Zeitersparnis : Jedes Cellophanblatt wurde ca. 1/2 Std. nach Aufbringen des Spritzbelages mit der begifteten Schicht nach oben so auf die kreisförmige Öffnung (ca. 7,5 cm $\varnothing$ ) eines Polystyrolkästchens gelegt, daß die mit DiDiTan behandelte Kreisfläche gerade die Öffnung bedeckte. Ein auf der äußeren Fläche mit Leim (Pelikan, Gummi Gum) bestrichener Käfig. ring wurde leicht auf das behandelte Cellophan gedrückt. Dieses legte sich dem Ring an und klebte fest. Die überragenden Teile des Cellophans wurden abgeschnitten. Dasselbe Verfahren wurde für den Verschluß der anderen Seite des Käfigs wiederholt.

Zur Trocknung des Leimes und zur Verdunstung der Azetonreste verblieben die Käfige vor Einbringung der Versuchsbienen ca. 2 Std. in Raumtemperatur. Mit einer Nadelbürste perforierte ich die Cellophanflächen, um eine ausreichende Sauerstoffversorgung der Tiere sicherzustellen.

Die einmalige Benutzung des Kä figs schaltete Fehlerquellen durch ältere Giftspuren aus; zugleich ersparte sie umständliche Reinigungsarbeit.

\section{Bestimmung der Kontaktgiftwirkung}

Durch diese Versuchsanordnung sollte der Toxizitätsgrad des Präparates DiDiTan Ultra bei Bienen unterschiedlicher genetischer Konstitution und bei verschiedenen Umweltfaktoren ermittelt werden. Eine Voraussetzung war zunächst die Bestimmung der Giftmenge, die unter standardisierten Versuchsbedingungen eine 50\%ige Abtötung der Versuchstiere einer "Standardpopulation " in Dauerkontakt (Residualwirkung) erreicht.

In jedem Versuch wurden in der Regel 5 Konzentrationsstufen bei zehnmaliger Wiederholung, falls nicht anders angegeben, geprüft. Die Abstufung der Konzentrationen erfolgte zumeist nach dem von Beran et NeURURer (1955) und KосH (1959) verwendeten Verfahren der " Halbierungsreihen ».

Versuchsbienen eines bestimmten Alters sammelte ich von den Waben und setzte sie im Laboratorium sofort in die mit einer bestimmten Insektizidmenge versehenen Käfige. In jeden Käfig wurden durch die Seitenöffnung 10 Bienen unbetäubt eingebracht und mit Futter (50\%ige Zuckerlösung, mittels einer Injektionsspritze ins Röhrchen gesaugt) versorgt (Abb. 3).

Zur Kontrolle dienten ausschließlich mit Azeton in gleicher Weise behandelte Käfige. Mit wenigen Ausnahmen wurde in den Gift- und Kontrollversuchen die gleiche Anzahl von Bienen geprüft.

Die Käfige verblieben 24 Stunden lang bei der gewünschten Temperatur im Brutschrank. Nach dieser Zeit erfolgte eine Registrierung des Totenfalles. Auf die Einhaltung einer bestimmten rel. Feuchte im Innern des Brutschrankes wurde geachtet.

\section{Bestimmung der per os- Giftwirkung}

Die perorale Giftapplikation von Dipterex SP 80 erfolgte auf der Basis der von GoEtze (1932) veröffentlichten Methode (WeAwer, 1949; BeraN et NEURURer, 1955, Koch, 1959).

Die von den Waben des Pflegevolkes abgesammelten Bienen verblieben etwa 1 Stunde bei Zimmertemperatur ohne Futter, um das Futterbedürfnis zu steigern. Danach wurden sie unbetäubt einzeln in ein Glasröhrchen von etwa $70 \mathrm{~mm}$ Länge und $8 \mathrm{~mm}$ Durchmesser gebracht und erhielten individuell mittels einer geeichten Mikropipette die vorgesehene Giftmenge. Diese wurde in einer mit Aqua dest. hergestellten 50\%igen Zuckerlösung frisch angesetzt. Die Benutzung von Leitungswasser führte zu unkontrollierten Versuchsbedingungen. Auch hier wurde die gleiche Anzahl von Dosierungsstufen bzw. Wiederholungen und nach demselben Verfahren wie bei den Kontaktversuchen geprüft. Vor dem Beginn der Applikation bestimmte ich den $\mathrm{pH}$-Wert jeder Konzentrationsstufe.

Während der Fütterung aus der Mikropipette verblieb die Biene in dem Röhrchen. Die Aufnahme der süß schmeckenden Lösung erfolgte zumeist sehr schnell; so war die Verabreichung der Lösung an $80-100$ Bienen etwa 2 Stunden nach ihrem Ansatz beendet. Alle Tiere erhielten mit je $10 \mathrm{~mm}^{3}$ des Zuckerwasser-Giftgemisches ein für alle Dosierungen konstantes Flüssigkeitsvolumen. Daß die Bienen die aufgenommene Giftlösung erbrachen, wie BerAN und Neururer (1955) berichten, konnte ich nicht beobachten. Die gleiche Anzahl von Kontrollbienen erhielt bei sonst gleichartiger Behandlung nur eine 50\%ige Zuckerlösung.

Je 10 Tiere von den Gift- und Kontrollversuchen wurden in Holzkästchen getrennt untergebracht und, mit $50 \%$ iger Zuckerlösung als Futter, weiter wie bei den Kontaktversuchen im Brutschrank auf Verhalten und Überleben geprüft.

Während bei den Kontaktversuchen Bienen zwischen $5-12$ und $20-32$ Tage alt untersucht wurden, waren für die Fraßversuche nur Altbienen verwendbar, da die Jungbienen 
(bis zum 12. Tag nach meiner Versuchsmethode) bei der Applikation dieses Mittels in vorgegebener Zeit keine ausreichende Menge aufnehmen.

Um eventuelle Fehlerquellen auszuschließen, habe ich die zu vergleichenden Versuchsreihen zur selben Zeit angesetzt, falls es nicht anders erwähnt ist.

\section{c. - Versuchsauswertung}

Die Versuchsbienen wurden 24 Stunden nach der Giftapplikation kontrolliert und die Anzahl der toten registriert.

Für die Bestimmung des Toxizitätsgrades der beiden Mittel wählte ich die LD $_{50}$.

Die Auswertung der Versuche ist nach dem Verfahren der ( Probitanalyse ) durchgeführt worden (Bliss, 1935; FinNEY, 1952; WEBER, 1964). Dadurch kann man am besten die mittlere letale Dosis $\left(\mathrm{LD}_{50}\right)$ feststellen (vgl. auch Koch, 1959; Fink und HuNd, 1965; FrNk u.a., 1966)*.

Bei der Besprechung der Ergebnisse sind für jeden Versuch in den Tabellen die Regressionsgleichungen, die $\mathrm{LD}_{50}$ und die relative Wirksamkeit mit den $95 \%$-Vertrauensgrenzen, die $\mathrm{X}^{2}$-Werte für die Homogenität, Parallelität sowie relative Wirksamkeit und - soweit notwendig — die zugehörigen Freiheitsgrade angegeben.

Die Tabellen sind die Wiedergabe von Varianzanalysen, bei der ein totales $x^{2}$ folgendermaßen aufgegliedert wurde :

\begin{tabular}{|c|c|c|}
\hline & $\begin{array}{c}\text { FG } \\
\text { Degrés de liberté }\end{array}$ & $\begin{array}{c}\text { Bemerkungen } \\
\text { Observations }\end{array}$ \\
\hline 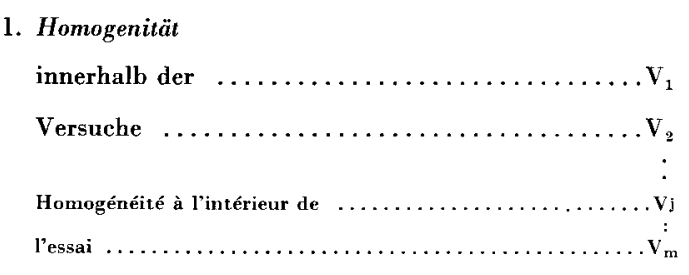 & $\begin{array}{c}\mathbf{n}_{1}-2 \\
\mathbf{n}_{2}-2 \\
\vdots \\
\mathbf{n}_{\mathbf{j}}-2 \\
\mathbf{n}_{\mathrm{m}}-2\end{array}$ & $\begin{array}{c}\mathbf{n}_{\mathbf{j}}=\text { Anzahl der } \\
\text { Dosierungen } \\
\text { Nombre des doses } \\
(\mathbf{j}=1,2, \ldots, m)= \\
\text { Index für den Versuch }\end{array}$ \\
\hline $\begin{array}{lc}\text { 2. Parallelität } & \begin{array}{c}\text { (Summe) } \\
\text { (Somme) }\end{array} \\
\text { Parallèlité }\end{array}$ & $\begin{array}{l}\qquad n_{j}-2 m \\
m-1\end{array}$ & \\
\hline $\begin{array}{lc}\text { 3. rel. Wirksamkeit } & \begin{array}{c}\text { (Summe) } \\
\text { (Somme) }\end{array} \\
\text { Activité relative }\end{array}$ & $\begin{array}{c}\ln _{j}-(m+1) \\
m-1\end{array}$ & \\
\hline $\begin{array}{c}(\text { Summe })=\text { Total } \\
(\text { Somme })=\text { Total }\end{array}$ & $\Sigma_{n}-2$ & \\
\hline
\end{tabular}

Die Versuche, bei denen für die Auswertung innerhalb jeder Dosis mit einer in der Regel aus 100 Bienen ermittelten Mortalität (in \%) gerechnet worden ist, sind aus $2-5$ Teilversuchen zusammengesetzt, die an verschiedenen Tagen durchgeführt wurden. Vor der Zusammen-

* Die Rechnungen wurden weitgehend unter Benutzung von zwei Computer-Programmen über "Probitanalyse " im Mathematisch-Naturwissensch. Institut, Abt. elektron. Datenverarbeitung, IBM7090/1410 - der Universität Bonn (Programm BMDO 3 S) und in dem Bayerwerk-Rechenzentrum, IBM-360/65 - Leverkusen durchgeführt. - Herrn Dr. Fink und Herrn Dipl. Mathem. Hund (Leverkusen) danke ich für ihre Hilfe. 
fassung wurden die Teilversuche nach dem oben angegebenen Schema der Varianzanalyse auf Übereinstimmung, d.h. auf Reproduzierbarkeit geprüft. Von wenigen Ausnahmen abgesehen, die dann von der weiteren Verrechnung ausgeschlossen wurden, war die Bedingung der Reproduzierbarkeit stets erfüllt. Die natürliche Mortalität der Bienen in den Kontrollversuchen lag mit wenigen Ausnahmen sehr niedrig; sie wurde deshalb in den Rechnungen nicht berücksichtigt.

\section{III. - ERGEBNISSE UND DISKUSSION}

Bei der Wiedergabe der Versuchsergebnisse in den Tabellen finden nachfolgende Symbole und Abkürzungen Verwendung :

u $=$ untere Grenzen

o $=$ obere Grenzen

$\mathbf{L D}_{50}=$ nach Individualanpassung

$+\quad=\mathrm{LD}_{50}$ nach Parallelanpassung

$\mathrm{R}=$ rel. Wirksamkeit (Numerus der Differenz der Logarithmen von $\mathrm{LD}_{50}$ nach Parallelanpassung)

Irrtumswahrscheinlichkeit in Prozent :

$>5=0$ (kein signifikanter Unterschied)

$5-1={ }^{*}$

$1-0,1=* *$

$<0,1={ }^{* * *}$

\section{a. - Kontaktversuche unter Berücksichtigung von :}

1. Alter

Die tatsächlich je Biene durch Kontakt bis zur Abtötung aufgenommene Giftmenge bleibt unbekannt. Eine exakte Berechnung ist nicht möglich, da die Giftaufnahme u.a. von der gleichmäßigen Verteilung, der Dicke der Schicht und der Bewegung der Bienen im Käfig abhängt (Beran et Neururer, 1955). Die Haltungsbedingungen der Bienen während der Versuchsdauer wie Raum, Futterangebot, Gasaustausch usw. können als geeignet betrachtet werden, da bei den Kontrollen keine oder nur eine sehr niedrige Sterblichkeit zu verzeichnen war.

Die Untersuchungen mit DiDiTan bei der Honigbiene ergaben, daß zwischen dem Alter der Tiere und der Wirksamkeit des Giftstoffes eine Abhängigkeit besteht (vgl. Tab. 2). Die Jungbienen waren empfindlicher als die älteren Bienen. Dieser Unterschied in der altersbedingten Empfindlichkeit konnte bei Bienen verschiedener Rassen und für zwei Temperaturstufen nachgewiesen werden. Auffallend ist die unterschiedliche Anfälligkeit von Jung- und Altbienen (Caucasica) bei $35^{\circ} \mathrm{C}$. Die $\mathrm{LD}_{50}$ wurde für die 5-9 Tage alten Bienen mit 216, $5 \mathrm{mcg}$ und für die 22-26 Tage alten Tiere mit $250,2 \mathrm{mcg}$ ermittelt. 


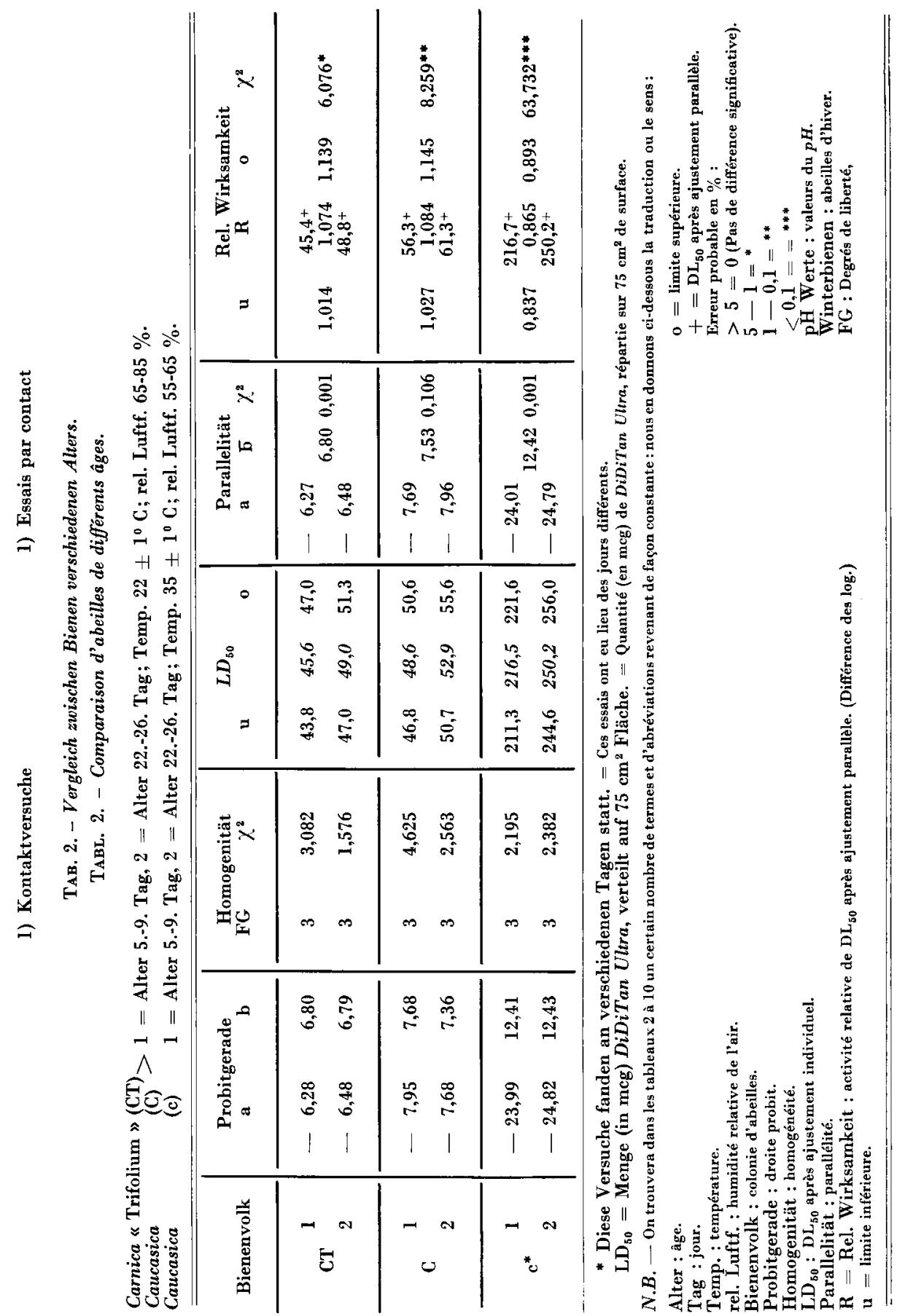


Unterschiedliche Empfindlichkeit bei Jung- und Altbienen gegenüber DDT haben auch Graves und Mackensen (1965) festgestellt. Obwohl das Alter der von ihnen als Stockbienen bezeichneten Versuchsgruppe unbekannt war, nehmen die Autoren an, daß die meisten getesteten Stockbienen älter als die eindeutig klassifizierten 2-8 Tage alten Tiere waren. Ihr Ergebnis, da $B$ die 8 Tage alten Bienen empfindlicher als die vom 4 . und 6 . Tag reagierten, ist offenbar auf die verwendete Methode zurückzuführen; die 8 Tage lang im Käfig eingesperrten Bienen erfuhren wahrscheinlich eine größere Schwächung als die nur 4 oder 6 Tage unter diesen Bedingungen gehaltenen Tiere. Die höchste Mortalität wiesen die 2 Tage alten Bienen auf.

Bei per os-Versuchen mit DDT (rein) ermittelte Koch (1959) unterschiedliche Toxizitätswerte bei Jung- und Altbienen. Die $\mathrm{LD}_{50}$ lag bei Bienen zwischen dem 5 . und 9 . Tag bei $5,9 \mathrm{mcg} /$ Biene, bei älteren Tieren (17. - 21. und 30. - 32. Tag) bei 11,2-12,1 mcg/Biene.

Für andere Insektenspecies hat man verschiedene Mechanismen der unterschiedlichen $D D T$-Resistenz bei Larven und Imagines angeführt: Vermindertes Eindringungsvermögen durch die stärker ausgebildete Cuticula bei Imagines (Böнм, 1951), Speicherung des DDT' in Fett- und Lipoidreserven des Körpers mit nachfolgendem fermentativem Umbau in ungiftige Dichlorphenylessigsäure $(D D E)$ oder Dichlordiphenyläthylen (DDA) (Zusammenfassung siehe Martin, 1967 ; KLein und Korte 1970).

Für Apis mellifica liegen bisher keinerlei physiologische Untersuchungen vor, die eine Erklärung der unterschiedlichen Empfindlichkeit von Jungbienen und älteren Imagines bei unterschiedlich hohen Temperaturen liefern.

\section{Rasse und Inzuchtgrad}

Wie aus den Tabellen 3 und 4 zu ersehen ist, wurden signifikant unterschiedliche Werte zwischen Bienen verschiedener Rassen bzw. Herkünfte festgestellt.

Bei einem Vergleich zwischen den Bienenrassen Carnica und Caucasica reagierten Carnica-Bienen empfindlicher gegenüber DiDiTan Ultra als die Caucasica-Bienen. Dieser Unterschied ist besonders deutlich bei der Temperatur von $35^{\circ} \mathrm{C}$ mit einer $\mathrm{LD}_{50}$ bei der Carnica-Rasse von $209,1 \mathrm{mcg}$ und $250,2 \mathrm{mcg}$ bei der Caucasica-Rasse. Eine erhöhte Widerstandsfähigkeit der Caucasica-Winterbienen gegenüber dem Präparat im Vergleich zu den Ligustica-Winterbienen war ebenfalls bei $22^{\circ} \mathrm{C}$ nachweisbar.

Die bisher dargestellten Versuchsergebnisse machen außerdem deutlich, daß die Umwelttemperatur und die Wirkung des Präparates DiDiTan Ultra auf die Honigbiene eng korreliert sind (Tab. 2 et 3 ). Während bei $22^{\circ} \mathrm{C}$ die $\mathrm{LD}_{50}$ zwischen 45-57 mcg schwanken, betragen sie bei $35^{\circ} \mathrm{C}$ 209-250 meg. Die für $35^{\circ} \mathrm{C}$ ermittelten Wirkungsgeraden verlaufen bedeutend steiler als die bei $22^{\circ} \mathrm{C}$, so daß keine rel. Wirksamkeit (Parallelanpassung) zustande kommt. 


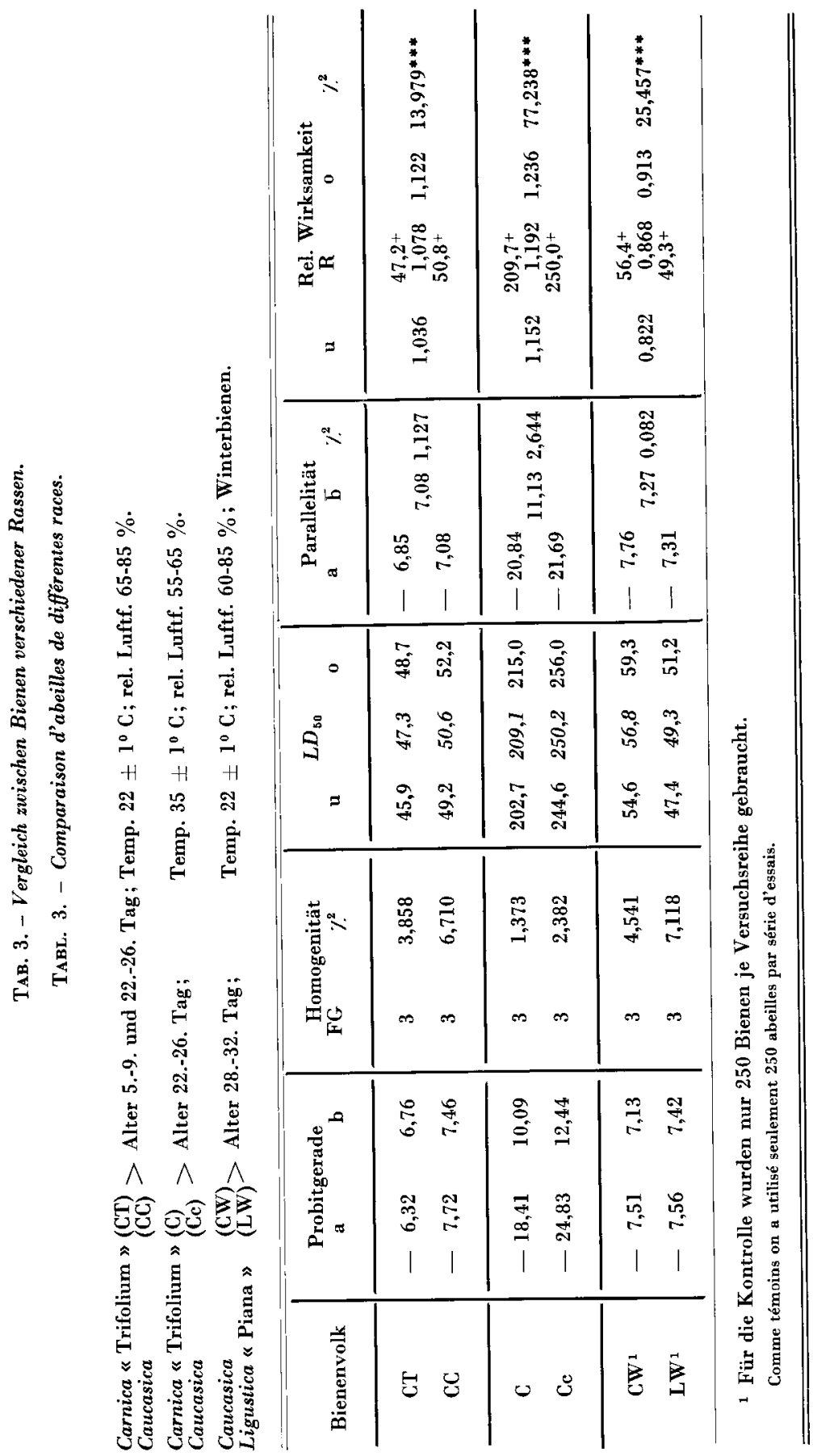




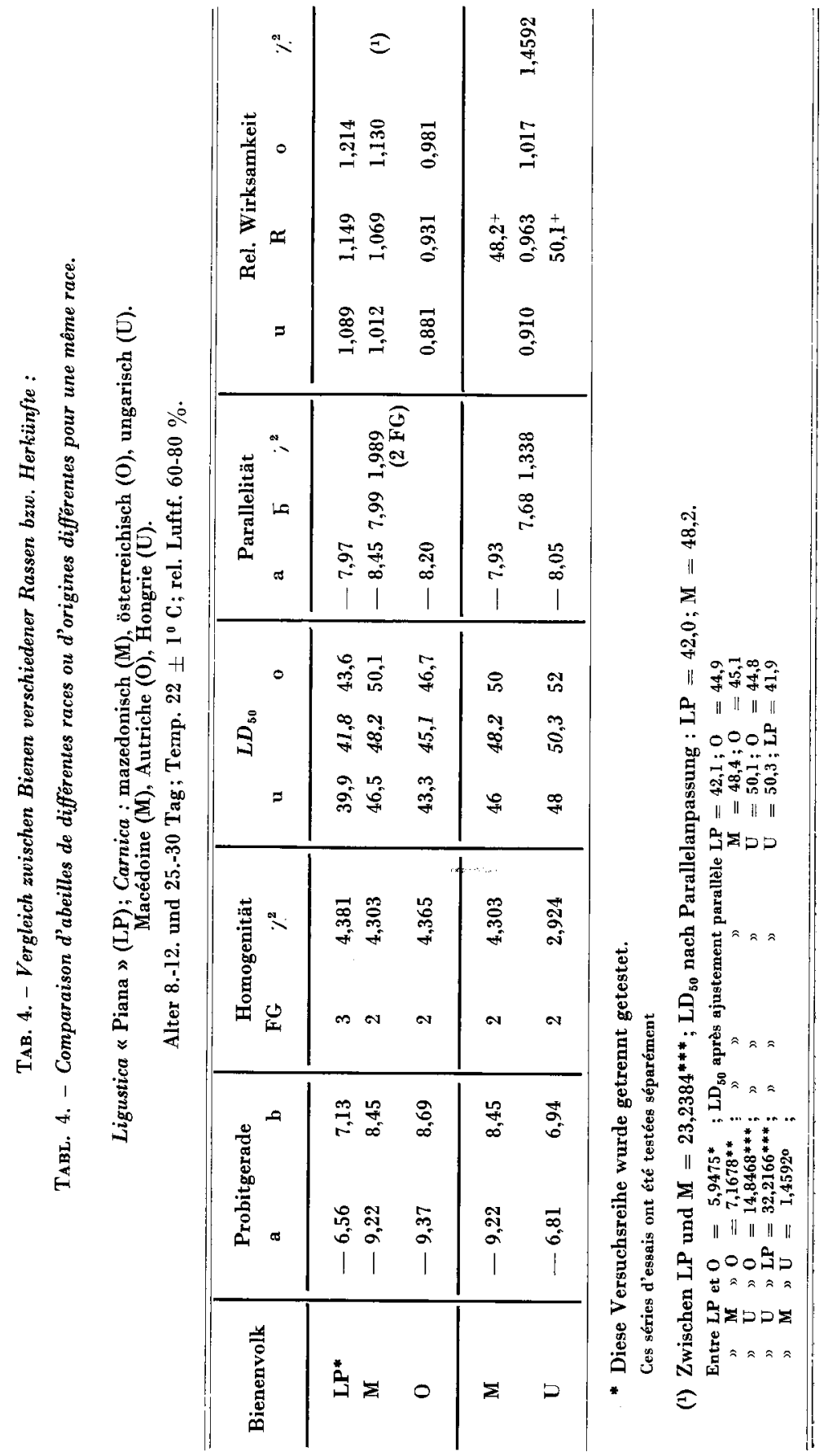


Die obigen Befunde bestätigen die Ergebnisse von HäFLIGER (1949, 1954), daß $D D T$ bzw. $D D T$-Präparate einen negativen Temperaturkoeffizienten besitzen, d.h. daß ihre Wirkung mit steigender Temperatur sinkt (KAESER, 1949 ; КосH, 1959).

Unterschiede konnte ich zwischen Ligustica und verschiedenen CarnicaHerkünften feststellen (Tab. 4). Ein Vergleich zeigt, daß die für diesen Versuch zur Verfügung stehenden Carnica-Bienen ungarischer und mazedonischer Herkunft widerstandsfähiger sind als die österreichischer Herkunft. Zwischen ungarischen und mazedonischen Carnica-Bienen konnte kein signifikanter Unterschied ermittelt werden. Merkliche Unterschiede bestanden dagegen zwischen den Carnica-Herkünften und Ligustica. Bienen dieser Rasse zeigten die höchste Empfindlichkeit gegenüber DiDiTan Ultra.

Aus den bisherigen Versuchsergebnissen wird außerdem deutlich, daß die Caucasica-Bienen eine höhere Toleranz gegenüber dem Präparat aufweisen als die Tiere der Carnica- und Ligustica-Rasse. Dieser Tatbestand wird in der Literatur als (" rassenspezifische Ansprechbarkeit ) bezeichnet und spielt vor allem eine besondere Rolle für die Prüfung von Pflanzenschutzmitteln auf ihren Toxizitätsgrad (UNTerstenhöfer, 1970).

Möglicherweise hat u.a. die unterschiedliche Körperkonstitution der Bienen zu diesem Ergebnis beigetragen; denn die Caucasica-Bienen waren größer und schwerer als die Carnica- bzw. Ligustica-Bienen. Stichproben von jeweils 10 vergifteten Bienen aus diesen Rassen ergaben, daß die CaucasicaBienen ein um $10-20 \%$ höheres Gewicht als die Tiere der Carnica- bzw. Ligustica-Rasse hatten.

Mit der Frage, ob zwischen Giftmenge und Körpergewicht der Insekten eine Beziehung besteht, haben sich Beran (1953) und WAY (1954) befaßt. BERAN (1953) stellte nach DDT-Dauerkontaktbehandlung bei der Hausfliege fest, daß mit steigendem Gewicht der Fliegen die Mortalitätsquote abnahm. Nach WAY (1954) stieg die Widerstandsfähigkeit der Larven von Diataraxia oleracea L. nach DDT-Behandlung mit Verdoppelung des Gewichtes auf das 11 bis 12 fache.

Zwischen den von mir und den von BeraN und Neururer (1955) in ihrem Cellophankäfig-Test festgestellten Werten besteht trotz der verschiedenartigen Methoden im allgemeinen eine gute Übereinstimmung.

Da Inzucht im allgemeinen mit einer verminderten Vitalität der Organismen gekoppelt ist, wurde auch hier die Widerstandsfähigkeit von zwei ingezüchteten Bienen-Linien (Carnica) und ihrem Kreuzungsprodukt (Bienen der 1. Generation) gegenüber DiDiTan Ultra geprüft (Tab. 5).

Die untersuchten Bienen der Kreuzungs-Generation $(F=0,03$ ) reagierten gegenüber dem Präparat gleichsinnig wie die des Vatervolkes $(F=0,25)$, die Tiere dieser beiden Völker waren widerstandsfähiger als die Bienen des anderen Elternteils (Muttervolk $F=0,25$ ) ; die ermittelten $L_{50}$ liegen jedoch auf der 


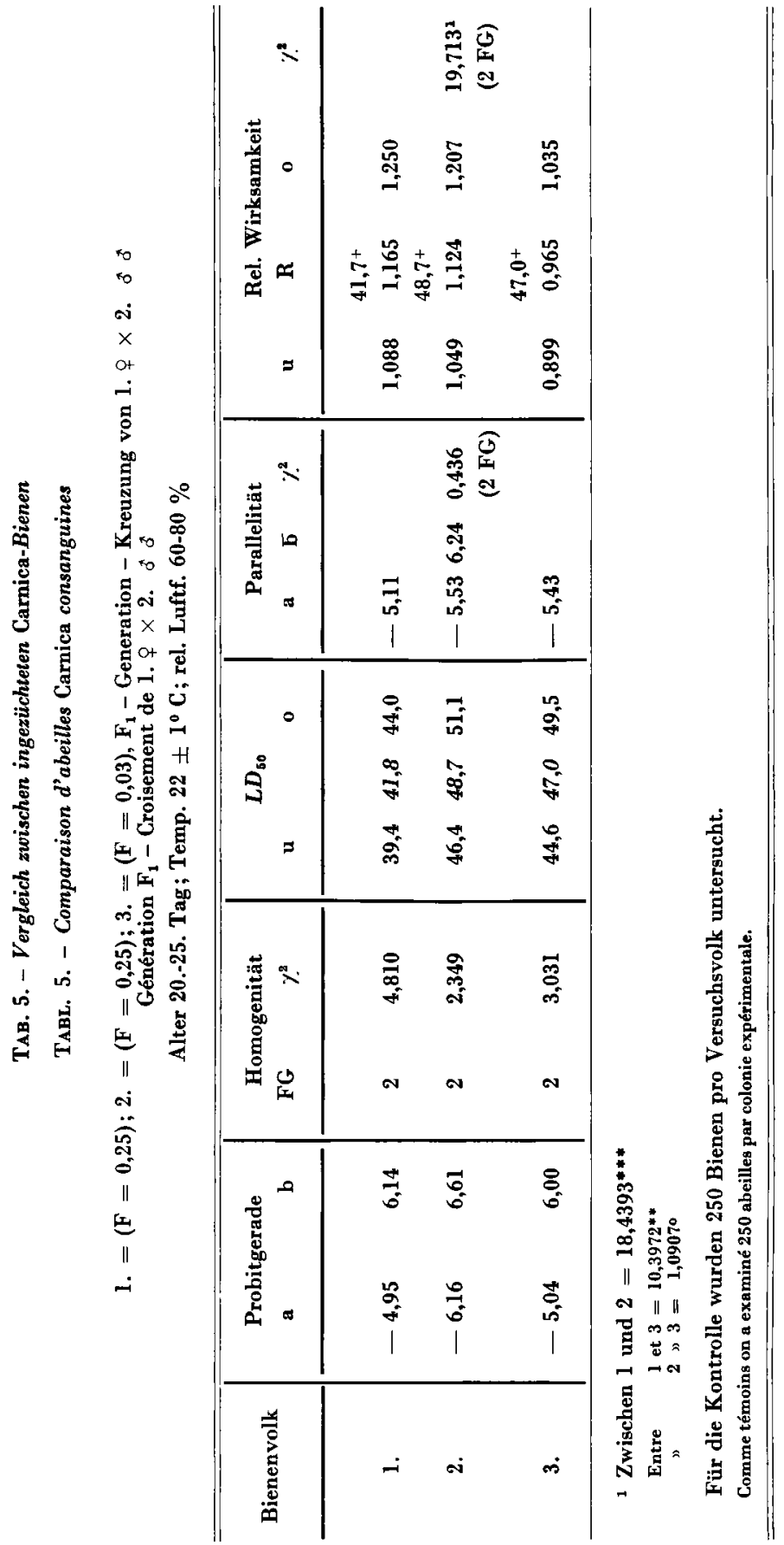



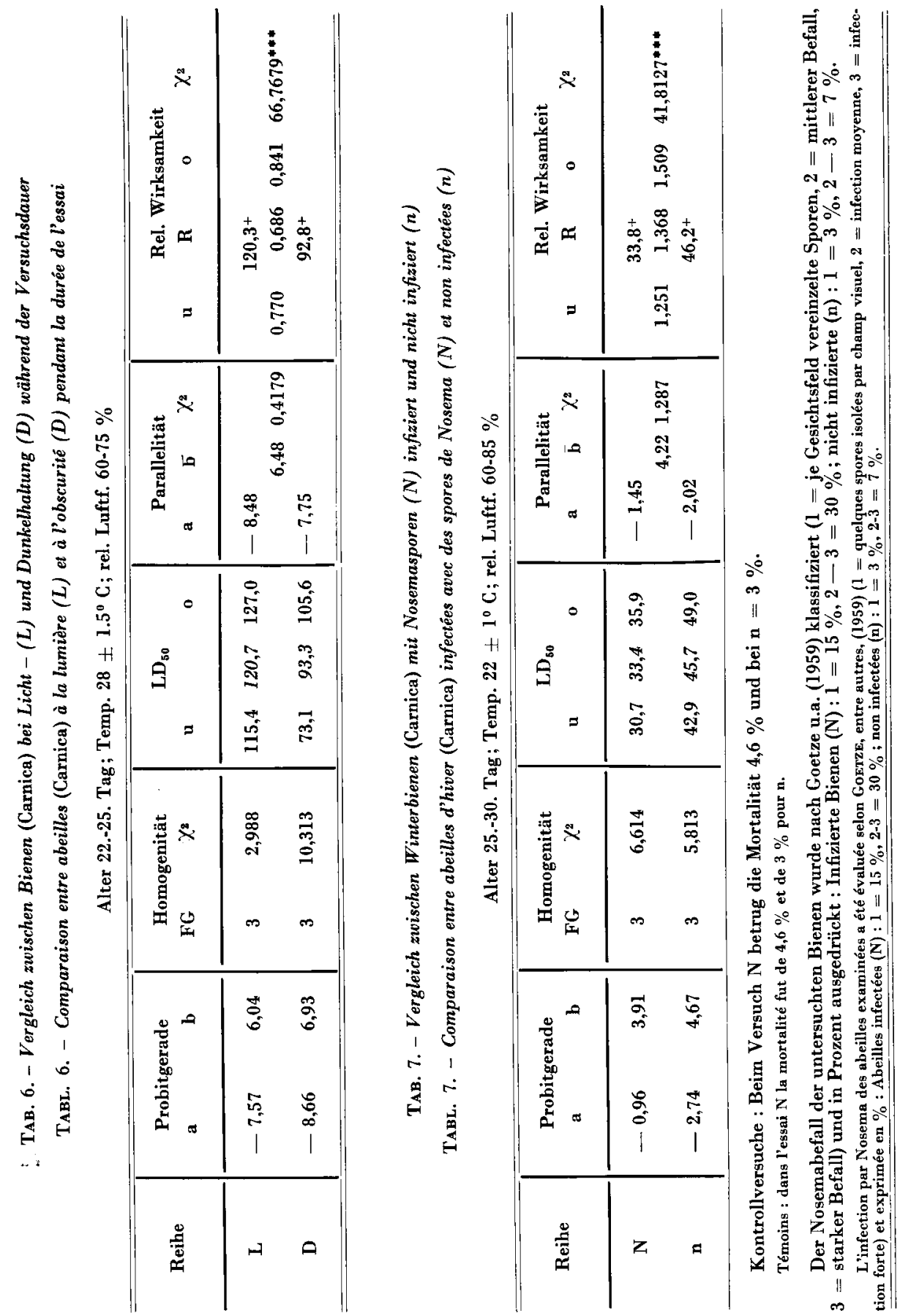
gleichen Ebene wie bei den vorstehend genannten untersuchten Bienenvölkern.

\section{Licht- und Dunkelhaltung}

Es ergab sich eine signifikant höhere Mortalität, wenn die Tiere während der Versuchsdauer bei Dunkelheit gehalten werden (Tab. 6). Vermutlich beruht dieser Unterschied darauf, daß die Bienen bei Licht aktiver waren und somit einen erhöhten Stoffwechsel hatten.

Die insektizide Wirkung von $D D T$ ist eng mit der Umwelt- und Körpertemperatur der Insekten verbunden (HÄFLIGER, 1948). Nach Angaben mehrerer Autoren zeigt $D D T$ - wie erwähnt - einen negativen Temperaturkoeffizienten bei Insekten (HäfLIGer, 1949, 1954; KAEsER, 1949 ; Guthrie, 1950 ; TAhori und Hoskins, 1953 ; Косн, 1959).

Wahrscheinlich entwickeln die Bienen im Licht infolge gesteigerter Aktivität eine höhere Körpertemperatur als Tiere bei Dunkelhaltung. Die Toleranz größerer Giftmengen ist die Folge.

Laboratoriumsversuche über die Wirkung von Insektiziden auf die Honighiene in künstlichem Licht sind mir nicht bekannt. Mehrere Autoren haben dagegen die Lichtverhältnisse und die Insektizidwirkungen auf andere Insektenarten untersucht.

LiNDQUist et al. (1946) stellten fest, daß sowohl UV-Licht als auch Sonnenlicht die Wirksemkeit von $D D T$ gegen die Hausfliege herabsetzte. Andere Forscher berichten hierzu, daß eine verminderte $D D T$-Wirkung bei Sonnen- bzw. UV-Licht-Exposition eintritt (Chisholm u. KoвLItsкy, 1947 ; Chisholm et al. 1949 ; Fleck, 1949).

Versuche unter Anwendung mehrerer Pflanzenschutzmittel in künstlichem Licht gegen den Baumwollkapselkäfer (Anthonomus grandis) haben amerikanische Autoren durchgeführt. Sie fanden die Insektizidwirkung von Parathion gegen diesen Schädling im Licht niedriger als in Dunkelheit. Die Toxizität von Parathion war von der Lichtintensität abhängig. Sie nahm mit zunehmender Helligkeit ab und erreichte bei völliger Abdunkelung ihr Maximum (Drössmar, 1965 ; Mistric und Gaines, 1953).

\section{Nosemainfeltion}

Ein enger Zusammenhang zwischen der Wirkung von DiDiTan Ultra und dem physiologischen Zustand der Bienen ließ sich im Versuch mit den mit Nosema infizierten und nicht infizierten Tieren nachweisen (Tab. 7). Die mit Nosema infizierten Bienen waren emp findlicher als die nicht infizierten. Die $\mathrm{LD}_{50}$ betrug bei ersteren $33,4 \mathrm{mcg}$ und bei den nicht in fizierten Tieren 45, 7 .

Auffallend bei den Versuchsen mit nosemainfizierten Bienen war die höhere Sterblichkeit der Kontrolltiere (Tab. 7). Dies ist offensichtlich mothodisch bedingt, da die Tiere 5-8 Tage im Brutschrank gehalten und nur mit Zuckerwasser versorgt wurden. 
Stute (1966) stellte fest, daß zahlreiche eingeschickte Proben von vergifteten Bienen zugleich Nosemabefall aufwiesen. Im allgemeinen ist, wie ThIEM (zit. Eichler, 1965) und Meyerhof (zit. Eichler. 1965) ausführen, eine Giftanfälligkeit der Honigbiene parallel zu ihrem Nosema-Infektionsgrad anzunehmen.

\section{b. - Fütterungsversuche, unter Berücksichtigung von :}

\section{Rasse}

Während bei den Kontaktversuchen nur ein « qualitativer » Vergleich von Bienengruppen gegenüber dem in ihrem Milieu diffus angebotenen Präparat möglich war, konnte bei oraler Applikation die Wirkung des Giftes bei der einzelnen Honigbiene in Korrelation zur aufgewendeten Menge genau bestimmt werden.

Im Verlauf meiner Untersuchungen zeigte sich, daß die Wirkung von Dipterex $S P 80$ bei der Biene eng mit dem $\mathrm{pH}$-Wert der verabreichten Lösung bei definierter Giftkonzentration verbunden war.

Fraßversuche bei Gruppen von Carnica-Bienen (20.-25. Lebenstag), denen 1,5 meg Dipterex $S P 80$ je Biene innerhalb von 60 Minuten nach Bereitung der Lösung mit Leitungswasser verabreicht wurden, führten zu folgendem Ergebnis :

Fraßversuche mit Dipterex SP 80 bei Carnica-Bienen (20. - 25. Tag.)

Versuchsdauer : 24 Std.; Temp. $22^{\circ} \mathrm{C}$; rel. Luftf. $60-80 \%$

\begin{tabular}{|c|c|c|c|}
\hline $\begin{array}{c}\text { Versuch } \\
\text { Essai }\end{array}$ & $\begin{array}{c}\text { Anzahl } \\
\text { gefütterte Bienen } \\
\begin{array}{c}\text { Nombre des abeilles } \\
\text { nourries }\end{array}\end{array}$ & $\begin{array}{l}\text { Darbietungszeit in Minuten nach } \\
\text { Ansetzen der Lösung } \\
\text { Temps (en minutes) entre la préparation } \\
\text { de la solution et sa présentation aux abeilles }\end{array}$ & $\begin{array}{l}\text { tote Bienen } \\
\text { Abeilles mortes }\end{array}$ \\
\hline 1. & $\begin{array}{l}20 \\
20 \\
20\end{array}$ & $\begin{array}{l}1 .-19 . \\
20 .-39 . \\
40 .-60 .\end{array}$ & $\begin{array}{r}6 \\
13 \\
18\end{array}$ \\
\hline 2. & $\begin{array}{l}20 \\
20 \\
20\end{array}$ & $\begin{array}{l}1 .-19 . \\
20 .-39 . \\
40 .-60 .\end{array}$ & $\begin{array}{r}3 \\
11 \\
18\end{array}$ \\
\hline
\end{tabular}

Es liegt somit eine Korrelation zwischen zunehmender Mortalität der Bienen und der Darbietungszeit der Dipterex-Lösung nach ihrer Bereitung vor. Dieses Phänomen läßt sich durch die chemischen Eigenschaften des Mittels erklären. Der Wirkstoff ist im Wasser instabil und setzt sich leicht in die verwandte Verbindung “ Dichlorphos 》 (DDVP) um, die erheblich giftiger für die Biene ist als Dipterex (Beran, 1965; Anderson und Atkins, 1966).

Wenn die gleiche Applikationsmenge (1,5 mcg/Biene) den Bienen 2 und 
mehr Stunden nach Bereitung der Lösung zugeführt wurde, ergab sie innerhalb weniger Stunden eine $100 \%$ ige Mortalität der Tiere. Danach kann die Hauptursache der grossen Differenz zwischen den Ergebnissen anderer Autoren (Maurizio u. Schenker, 1957; Beran, 1958) in den unterschiedlichen pHWerten bzw. in unterschiedlichem Alter der applizierten Lösung gesucht werden.

Diese Umlagerung in wässriger Lösung verursachte auch wohl die unterschiedlichen Glashaus-Versuchsergebnisse mit Dipterex auf die Honigbiene (Beran und Neururer, 1956).

Per os-Versuche mit Nektar, der aus mit Dipterex behandelten Pflanzen (Borago officinalis) gewonnen wurde, zeigten nach Maurizio und Schenker (1957) eine sehr hohe Bienentoxizität bis 48 Stunden nach der Pflanzenbehandlung.

Andere Autoren (Unterstenhöfer, 1957 ; Anderson et Atkins, 1958) betrachten Dipterex als nicht gefährlich für die Biene. Diese Auffassung ist aber nur dann gültig, wenn das Mittel als Kontaktinsektizid Verwendung findet. Ich konnte unter ständiger Kontrolle des $\mathrm{pH}$-Wertes der verabreichten Insektizid-Lösung den toxischen Effekt des Präparates bei Bienen unterschiedlicher genetischer Herkunft und bei bestimmten Umweltbedingungen ermitteln (Tab. 8).

Wie aus der Tabelle ersichtlich ist, waren die in meinem Versuch verwendeten Caucasica-Bienen widerstandsfähiger als die Carnica-Bienen. Dieser Unterschied zeigte sich eindeutig bei Temperaturen von $22^{\circ} \mathrm{C}$ und $35^{\circ} \mathrm{C}$.

Beim Vergleich der ersten zwei Versuchsreihen der Tabelle ist festzustellen, daß (mit kleiner Differenzierung der untersuchten $\mathrm{pH}$-Wert-Bereiche der applizierten Giftlösung) die Wirksamkeit des Präparates von der Temperatur abhängt. Die mittlere letale Dosis (mcg pro Biene) beträgt bei $35^{\circ} \mathrm{C}$ bei den Carnica-Bienen 3,2 und bei den Caucasica-Bienen 4,3. Sie ist bei $22^{\circ} \mathrm{C}$ mit 6,3 bei Carnica-Bienen und mit 7 bei Caucasica-Tieren etwa doppelt so hoch.

Im Gegensatz zu DiDiTan Ultra besitzt Dipterex SP 80 also einen positiven Temperaturkoeffizienten.

Signifikant ist auch die Differenz zwischen der Sterblichkeitsrate bei Caucasica- und Ligustica-Winterbienen (Tab. 8). Die Caucasica-Bienen vertrugen etwa die doppelte Giftmenge wie die Ligustica.

\section{Licht-und Dunkelhaltung}

Tab. 9 zeigt die erhöhte Giftmenge, die zur Erzielung des gleichen Effektes bei Licht erforderlich war.

Wie auch bei den Kontaktversuchen nahmen die Bienen bei Licht mehr normales Futter auf als die Tiere im abgedunkelten Brutschrank.

Eine derartige Herabsetzung der toxischen Wirkung durch Haltung der 


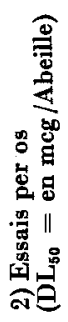

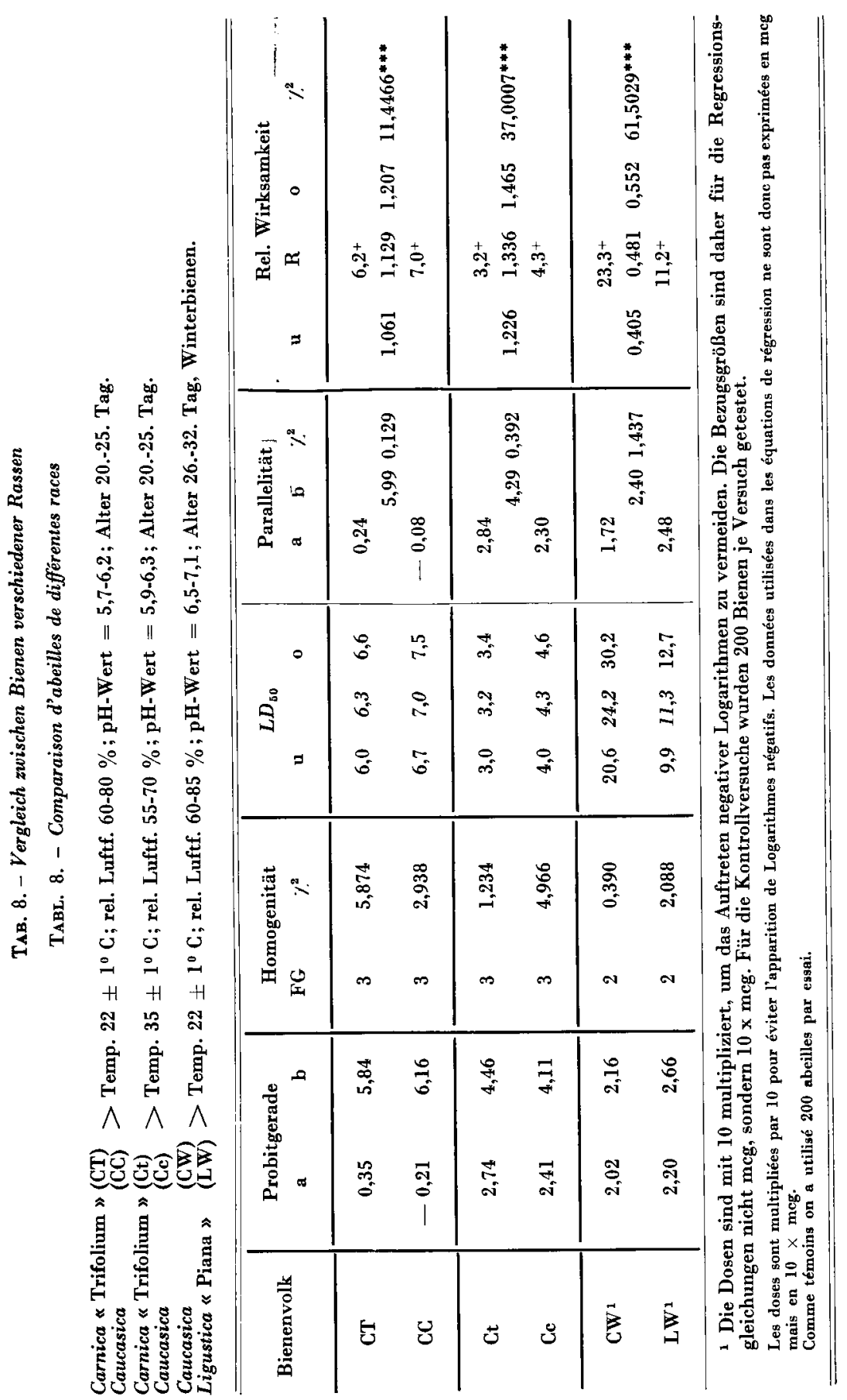


ANFÄLLIGKEIT GEGENÜBER ZWEI INSEKTIZIDEN
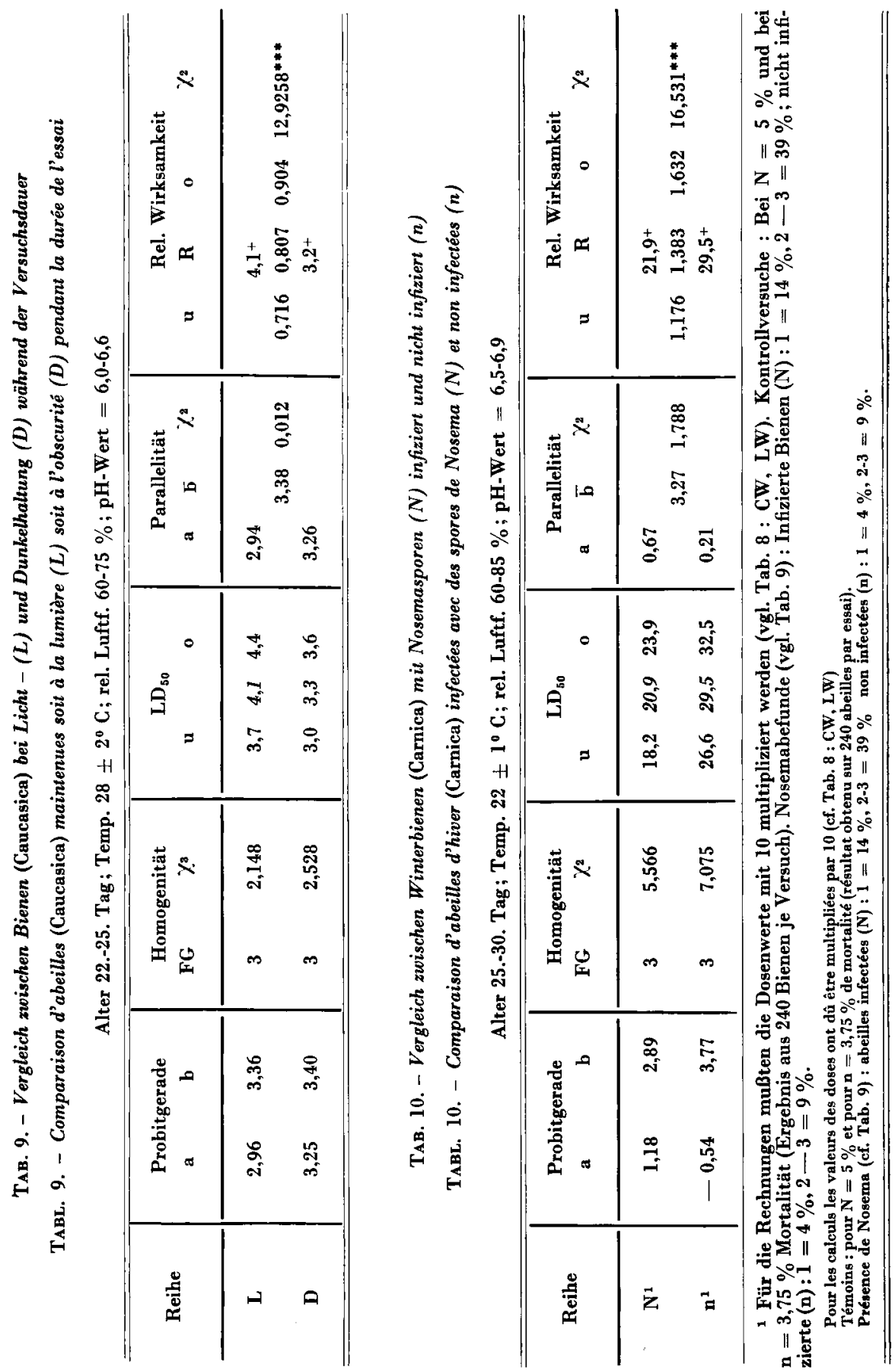
Tiere im Licht nach der Giftapplikation wurde bei verschiedenen Insektiziden und bei anderen Insektenarten nachgewiesen (s.S. 13).

\section{Nosemainfektion}

Die Giftdosis $\left(\mathrm{LD}_{50}\right)$ von Dipterex $S P 80$ bei Bienen, die mit Nosemasporen infiziert waren, lag niedriger als bei den nicht infizierten, wie aus Tabelle 10 ersichtlich ist. Dieser Unterschied spricht für eine erhöhte Anfälligkeit von nosemainfizierten Bienen gegen Fraßgifte aus der Phosphorsäureestergruppe.

Wie bei den Kontaktversuchen führten auch hier methodische Gründe zu einer erhöhten Mortalität bei den Kontrollbienen (vergl. Tab. 7).

Eingegangen im Juni 1971.

Reçu pour publication en juin 1971.

\section{RÉSUMÉ}

Bien qu'une méthode normalisée soit utilisée pour tester la toxicité des produits phytosanitaires à l'égard de l'abeille, on constate souvent que les répétitions donnent des résultats divergents. Afin de déterminer l'influence de facteurs qui ne sont pas pris en considération par la méthode standard on a étudié : l'âge des abeilles, la race et le degré de consanguinité, l'éclairement et l'infection par noséma. Pour mettre en évidence une éventuelle résistance différentielle on a utilisé comme poison de contact le DiDiTan Ultra (DDT) et comme poison par ingestion le Dipterex (ester phosphorique). L'interprétation des résultats - influence des facteurs énumérés ci-dessus sur la D.L. ${ }_{50}$ - a été faite au moyen de l'analyse probit. Pour une application plus exacte du poison de contact on a mis au point une cagette monoservice composée d'un anneau de matière plastique et de feuilles de cellophane (Fig. 1-4). L'utilization per os du Dipterex a été faite par nourrissement individuel d'abeilles âgées de plus de 10 jours.

Le tableau 2 montre l'influence de l'âge des abeilles expérimentales sur leur sensibilité au poison. Les jeunes abeilles sont plus sensibles que les abeilles âgées; ceci est valable en général pour plusieurs races étudiées. Très net également est le coefficient de température négatif du DDT; lorsque la température augmente la toxicité diminue. Si on compare entre eux des représentants de plusieurs races, on constate que les caucasiennes sont les plus résistantes (Tableau 3). Dans ce cas il se peut que le phénomène soit en rapport avec le poids individuel généralement plus élevé des abeilles caucasiennes. A l'intérieur même d'une race - on a étudié des carnioliennes de trois origines géographiques différentes - on peut mettre en évidence une sensibilité différentielle (Tableau 4). Les connaissances antérieures sur l'action du Dipterex ont été confirmées (Tableau 8).

On sait que, fréquemment, la consanguinité est liée à une diminution de la vitalité. Une comparaison de deux lignées consanguines $(F=0,25)$, développées à partir du même matériel de départ, a montré une différence significative de la résistance de ces deux lignées (Tableau 5). Les abeilles de la génération $F_{1}$ après croisement $(F=0,03)$ correspondaient, par leur moins grande sensibilité, à la lignée paternelle, laquelle se situait dans le domaine normal des colonies carnioliennes non consanguines. 
Lorsque les abeilles étaient maintenues à l'obscurité après contact avec le DDT leur DL $_{50}$ était plus basse que lorsqu'elles étaient maintenues à la lumière (Tableau 6). L'activité accrue pendant l'éclairement augmentait probablement la température du corps ce qui avait pour conséquence de permettre la manifestation du coefficient de température négatif du DDT. L'éclairement ou l'obscurité après le nourrissement au Dipterex montraient un effet semblable (Tableau 9).

Le fait qu'on mette fréquemment en évidence une infection par nosema chez les abeilles intoxiquées laisse présumer que cette infection influence défavorablement la résistance aux toxiques. Les tableaux 7 et 10 démontrent l'abaissement de la $D_{50}$ chez les abeilles infectées artificiellement lorsqu'on les soumet à l'action du DDT et du Dipterex.

Une observation effectuée sur l'utilisation du Dipterex mérite encore d'être signalée. La toxicité de ce produit dépend de façon décisive de la valeur du $\mathrm{pH}$ du milieu aqueux dans lequel il est employé ainsi que du temps qui s'écoule entre la confection de la solution et son utilisation. La toxicité du Dipterex est plus élevée en milieu alcalin qu'en milieu acide; la toxicité augmente avec le vieilissement de la solution (Voir le tableau page 18, expériences de nourrissement).

Les résultats ci-dessus exposés donnent une indication sur les causes des différences obtenues dans les tests de toxicité de produits phytosanitaires, même lorsqu'une méthode standard est utilisée.

\section{LITERATURVERZEICHNIS}

Anderson L.D., Atkins E.L., 1958. Toxicity of Pesticides to Honey Bees in Laboratory and Field in Southern California. J. of Econ. Entom., 51, 103-108.

1965. Research on the Effect of Pesticides on Honey Bees. Amer. Bee J., 106, 206-208, 1966.

Pesticides usage in relation to beekeeping. Ann. Rev. Entom., 13, 213-238, 1968.

Atkins E.L., Anderson L.D., 1962. DDT resistance in honey bees, J. Econ., Entom., 55, $791-92$.

Beran F., Neuruner J., 1955. Zur Kenntnis der Wirkung von Pflanzenschutzmitteln auf die Honigbiene (Apis mellifica L.) 1. Mitteilung. Bienengiftigkeit von Pflanzenschutzmitteln. (Wien) Pflanzenschutzberichte, 15, 98-147.

Buss C.I., 1935. The calculation of the dosage mortality curve. Ann. Applied Biol., 22, 134167.

Chisholm RD., Koblitsky L., 1947. Effect of light on DDT residues, Agric. Chemicals, 2 (9), 35-37.

Chisнolm et al., 1949 The toxicity of DDT deposits as enfluenced by sunglibt. J. Econ. Entomol., 42, 154-55.

Drössmar F., 1965. Lichtabhängige Wirkung von Strahlen und chemischen Substanzen,

Naturwiss. Rundschau, 52, Heft 11, 456.

EICHLER W., 1965. Handbuch der Insektizidkunde, Verl. Volk und Gesundheit - Berlin.

Fivk H., Hund G., 1965. Probitanalyse mittels programmgesteuerter Rechenanlagen, Arzneim.-Forsch. (Drug Res.), 15, 624-30.

FINK H., u.a., 1966. Vergleich biologischer Wirkungen mittels programmierter Probitanalyse, Sonderdruck - Methods of Information in Medicine - Methodik der Information in der Medizin. 5. Jahrg. (1), 19-25, F.K. Schattauer-Verl., Stuttgart.

Finney D.J., 1952. Probit Analysis, 2. Edition, Cambridge.

FLECK E.E., 1949, The action of ultraviolet light on DDT $J$. Amer. Chem. Soc., 71, 1034-1036.

Fucuto T.R., 1961. The Chemistry of Organic Insecticides. Annual Rev. Entomol., 6, 313-332.

GaInes J.C., Dean H.A., 1950. Effect of climatic factors on the toxicity of certain insecticides. J. Econ. Entomol., 43, 602-605.

Goetze G., 1932. Zwei neue Methoden zum quantitativen Studium der Giftigkeit von Insektiziden, Anzeiger f. Schädlingskunde, 8, 54-57.

Goetze G., u.a., 1959. Versuche zur Selbstheilung und Therapie der Nosematose der Honigbiene, Bonn.

Graves J.B., Mackensen O., 1965. Topical Application and Insecticide Resistance Studies on the Honey Bee. U. Econ. Entomol., 58, 990-993. 
Guthrie F.E., 1950. Effect of Temperature on Toxicity of Certain Organic Insecticides. $J$. Econ Entomol., 43, 559-60.

Hafliger E., 1948. Der Einfluß der Temperatur auf die Giftwirkung des DDT bei Honigbienen (Apis mellifica L.) Experientia, 4, 223-224.

1949. Comparative Toxicity of Various Insecticide to the Honeybee. J. Econ. Entomol., 42, 523-528.

1954. Der Einfluß der Temperatur auf die Wirkung von DDT-Präparaten, Z. f. P flanzenkrankheiten und Pflanzenschutz 61, 433-444.

KaEser W., 1948. Zur Frage einer temperaturbedingten Widerstandsfähigkeit der Honigbiene (Apis mellifica L.) gegenüber dem Kontaktinsektizid (Gesarol). Anzeiger f. Schädlingskunde 21, 129-132.

Косн H., 1959. Über die Giftwirkung der beiden Insektizide Toxaphen und DDT auf verschiedene Imaginalstadien der Honigbiene (Apis mellifica L.) und ihre Abhängigkeit von der Temperatur. Wiss. Zeitschr. der Karl-Marx-Univers. Leipzig, Mathem.-Naturwiss. Reihe, Heft 1, 8. Jahrg., 203-215 $1958 / 1959$.

LindQuist A.W. u.a., 1946. DDT residual type sprays as affected by light. J. Econ., Entomol., 39, 55-59.

Maurizio A., Schenker P., 1957. Untersuchungen über die Ausscheidung bienengiftiger Pflanzenschutzmittel im Blütennektar, Mitteil. der Schweizer. Entomolog. Gesellschaft, 30, 140-150.

Mistric W. J., Gaines J.C., 1953. Effect of Wind and other Factors on the Toxicity of Certain Insecticides. J. Econ. Entomol., 46, 341-49.

Mühlmann R., Schrader G., 1957. Hydrolyse der insektiziden Phosphorsäureester, Z. Naturforschung, $12 \mathrm{~b}, 196-208$.

Schrader G., 1963. Die Entwicklung neuer insektizider Phosphorsäure-Ester, 3. Aufl., Verlag Chemie GmbH. Weinheim/Bergstr.

Stute K., 1964. Über die Wirkungsweise einiger bienenschädlicher Insektizide, Z. für Bienenforschung, $7,89-97,1964$.

1966. Bienenschutz - Pflanzenschutz. Westfälische Bienen-Zeitung, 79, Jahrgang, 304-6. 1969. Richtlinien für die Prüfung von Pflanzenschutzmitteln auf Bienengefährlichkeit, Bundesforschungsanstalt für Kleintierzucht, Celle, Januar 1969 (Sonderheft).

TahonI A.S., Hoskins W.M., 1953. The absorbtion, distribution and metabolism of DDT in DDT-resistant hous flies. J. Econ. Entomol., 46, 302-306 und 829-837.

Unterstenhöfen G., 1957. Dipterex, ein neues ungiftiges Insektizid. Anzeiger $f$. Schädlingskunde, 30, Jahrg., 7-10.

$1968 / 1$ : Die Bedeutung der organischen Phosphorverbindungen für den Pflanzenschutz. Pflanzenschutznachrichten Bayer, 21, Jahrg., 53-78.

1970. Allgemeines über Biologie und Prüfung der Insektizide und Akarizide, s. Wegler, R., Chemie der Pflanzenschutz- und Schädlingsbekämpfungsmittel, Bd.1, Springer-Verlag, Berlin, Heidelberg, New York., S. 57-76.

Unterstenhöfer G., Frehse H., 1963. Wesen und Bedeutung der systemischen Wirkung von Insektiziden. Pflanzenschutz-Nachrichten Bayer, 16, 165-181.

WAY M.J., 1954. The effect of body weight on the resistance to insecticides of the lastinstav larva of Diabrotica oleracea L. tho tomato moth. Ann. Applic. Biol., 41, 77-87.

Weaver N., 1949. The Toxicity of certain Organic Insecticides to Honeybees. J. Econ. Entomol., 42, 973-75.

Weber E., 1964. Grundriß der biologischen Statistik, 5. Auflage, VEB Gustav Fischer Verlag Jena. 